\title{
Cálculo de registros sintéticos de ingresos por cuenca propia de un sistema de presas de la región Noroeste de México, caracterizada por eventos invernales
}

\section{Synthetic Generation of Inflows in a Dam System of a Region Characterized by Extraordinary Winter Events}

\author{
R. Domínguez-Mora \\ Instituto de Ingeniería, UNAM. \\ Facultad de Ingeniería, UNAM. \\ E-mail:rdm@pumas.iingen.unam.mx \\ M.L. Arganis-Juárez \\ Instituto de Ingeniería, UNAM. \\ Facultad de Ingeniería, UNAM. \\ E-mail:MarganisJ@iingen.unam.mx
}

(Recibido: septiembre de 2007; aceptado: junio de 2008)

\section{Resumen}

\begin{abstract}
En este estudio se generaron muestras sintéticas de series mensuales de volúmenes de ingreso por cuenca propia de cuatro presas en las que se preserva la autocorrelación y la correlación cruzada de las series históricas. Se utilizó el método de Svanidze modificado con una variante en la selección aleatoria de años, considerando que los ingresos anuales por cuenca propia a cada una de las presas tuvieron una distribución afectada por los eventos de invierno. Los estadísticos media, desviación estándar, coeficiente de asimetría y coeficiente de autocorrelación, así como la correlación cruzada, fueron reproducidos satisfactoriamente.
\end{abstract}

Descriptores: Muestras sintéticas, eventos de invierno, método de Svanidze modificado, escurrimientos, autocorrelación, correlacióncruzada.

\section{Abstract}

In this paper synthetic monthly series of net basin inflows in four dams were generated; there are correlations and crossed correlations in historic series; Svanidze's modified method was applied, adding a different way in the random selection of years including the fact that the net basin inflows distribution was influenced by winter events. Statistics media, standard deviation, skew, autocorrelation and crossed correlation were successfully reproduced.

Keywords: Sinthetic series, winter events, Svanidze's modified method, inflows, autocorrelation, crossed correlation.

\section{Introducción}

En el Noroeste de la República Mexicana el régimen normal de escurrimientos tiene su máximo en los meses de verano, de julio a octubre, y valores mínimos en invierno (diciembre, enero y febrero). Sin embargo, ocasionalmente se presentan tormentas de invierno, conocidas en la región como equipatas, de tal magnitud que los escurrimientos mensuales que provocan son mucho mayores que la media en esos meses, e incluso, sensiblemente mayores que los de los meses de verano; es por eso que en el análisis y la generación de 
DOI: http://dx.doi.org/10.22201/fi.25940732e.2009.10n4.032

Cálculo de registros sintéticos de ingresos por cuenca propia de un sistema de presas de la región Noroeste de México, caracterizada por eventos invernales

series sintéticas de escurrimientos se debe tomar muy en cuenta esta característica.

En este estudio se generaron muestras sintéticas de volúmenes mensuales de ingreso por cuenca propia a cuatro presas situadas en la cuenca del río Yaqui, en el estado de Sonora. La región en estudio se muestra en la figura 1 y en la figura 2 con la configuración del sistema formado por las presas Lázaro Cárdenas (Angostura), Son., Abraham González, Chih., Plutarco Elías Calles (El Novillo), Son., y Álvaro Obregón (Oviachic), Son.

Se utilizó el método de Svanidze modificado (Domínguez et al., 2001,2002), (Svanidze, 1980), con una variante en la selección aleatoria de los años, debido a que en un ensayo preliminar se observó que el método en su forma original reportaba valores sintéticos de las asimetrías considerablemente mayores que los históricos (hasta más del doble), en prácticamente todos los meses. Lo anterior se atribuyó a la existencia de eventos extraordinarios de invierno en años con valores grandes del ingreso por cuenca propia. Las muestras generadas preservan satisfactoriamente las características estadísticas (media, desviación estándar y coeficiente de asimetría de cada mes) de los escurrimientos de cada una de las presas, la función de distribución de los escurrimientos anuales totales, los coeficientes de autocorrelación entre meses sucesivos y los coeficientes de correlación cruzada, para cada mes del año, entre cada pareja de presas.

\section{Desarrollo}

Método de Svanidze modificado en la selección aleatoria de años

En el análisis de series de tiempo, existen numerosas metodologías para obtener registros sintéticos a partir del registro histórico, tanto de series anuales como de series periódicas (semestrales, mensuales, diarias); entre esos métodos cabe destacar los modelos autorregresivos de promedios móviles ARMA (p,q) (Salas et al., 1988), modelos ARIMA y de Thomas Fiering (Yurekly et al., 2004), el método de Fragmentos de Svanidze (Svanidze,1980).

Los modelos ARMA tienen el inconveniente de que su aplicación se limita a series de datos cuya distribución debe ser normal y aunque existen técnicas para efectuar una transformación normalizante a la serie original, tales como el cálculo del logaritmo natural o el método de Box y Cox (Escalante, 2002), por tratarse de transformaciones no lineales, al aplicar la transformación inversa se incurre en una deformación de los resultados finales.

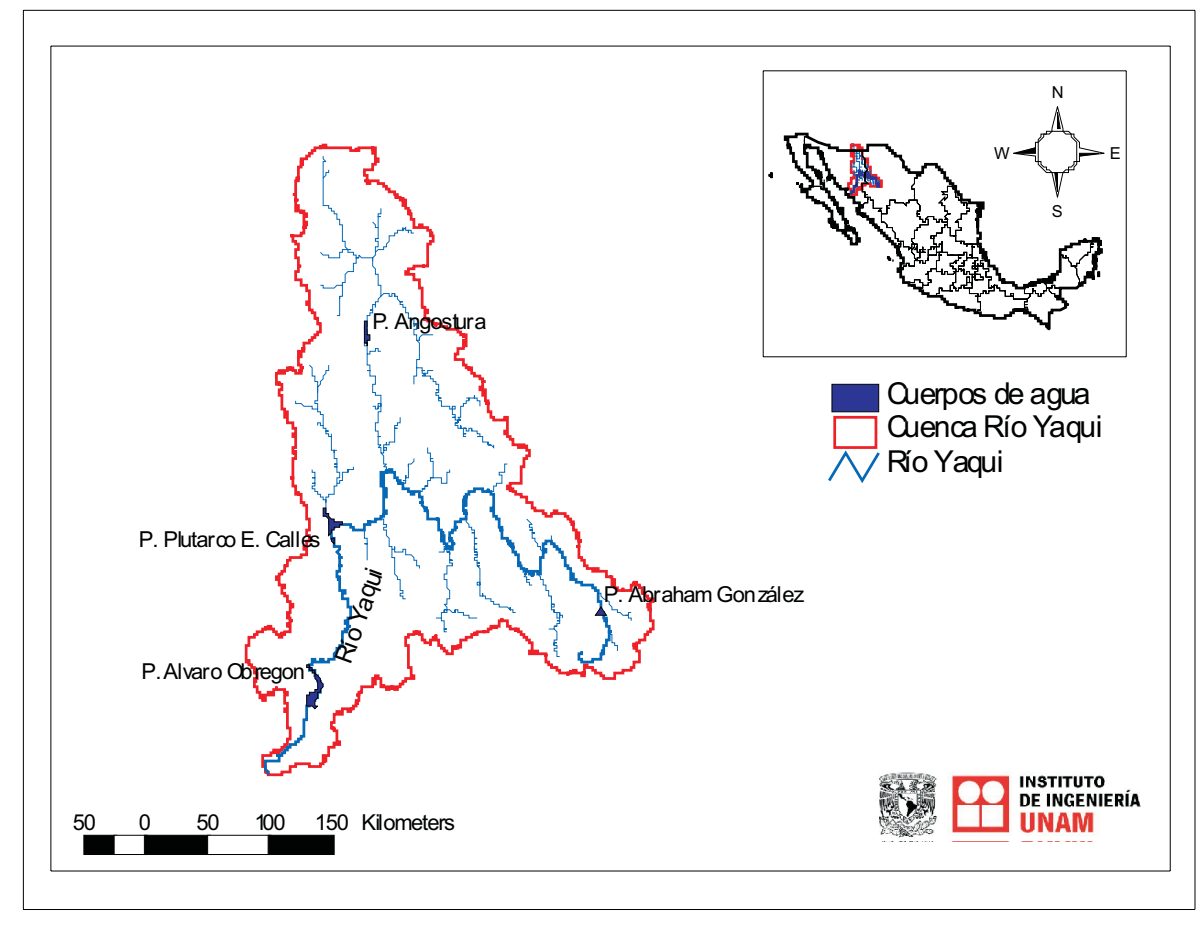

Figura 1. Localización del sitio, río Yaqui, Sonora, México 


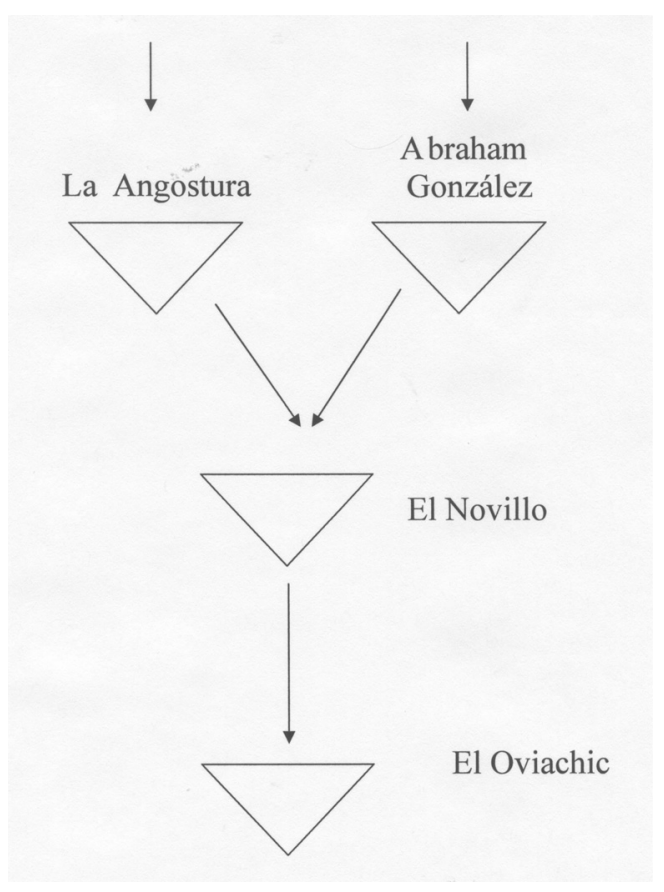

Figura 2. Configuración del sistema de presas

El método de Svanidze modificado, ha sido utilizado con éxito en la generación sintética de los volúmenes de escurrimiento de sistemas de presas que operan en cascada (Domínguez et al., 2001, 2002); tiene la ventaja de que no se requiere que los datos sean normales y que logra reproducir las correlaciones y correlaciones cruzadas. El problema que tiene es la preservación de la correlación entre el último periodo del año $i$ y el primer periodo del año $i+1$, pero lo anterior se resuelve identificando los periodos de más baja correlación y redefiniendo los años (en lugar de usar años cronológicos, se utilizan años hidrológicos).

Los datos de entrada al proceso son las $n$ series periódicas históricas analizadas. Para cada año del registro histórico se calculan los totales de cada serie y la suma de los $n$ totales; adicionalmente, para cada una de las series se obtiene la fracción del ingreso mensual con respecto al total anual y se determina, para cada total anual de cada serie, el porcentaje con respecto al total suma. A la serie anual formada por la suma de $\operatorname{los} n$ totales se le hace un análisis estadístico para determinar la función de distribución de probabilidades de mejor ajuste.

La generación sintética se realiza mediante un doble procedimiento aleatorio; el primero consiste en utilizar la función de distribución de mejor ajuste, con la que se obtienen $m$ valores aleatorios con la función de distribución de la suma de los $n$ totales. El segundo procedimiento es la selección aleatoria de entre $m$ años históricos para obtener el porcentaje sintético tanto de los totales anuales de cada serie, como las fracciones mensuales correspondientes. Se obtienen los totales anuales sintéticos para cada serie multiplicando el total suma aleatorio por el porcentaje correspondiente al año seleccionado aleatoriamente. Se multiplica el total anual sintético de cada serie por las fracciones mensuales correspondientes al año seleccionado, con lo que se determinan las $m$ series periódicas sintéticas.

\section{Modificación al algoritmo en la selección de los años aleatorios}

En el estudio de las presas del río Yaqui, al hacer la aplicación del método de Svanidze modificado con el procedimiento descrito anteriormente, no se lograron reproducir los coeficientes de asimetría (figura 3); la causa atribuida a este problema fue la existencia ocasional de valores registrados en los meses de invierno de magnitud considerablemente mayor a la media anual.

Considerando que los volúmenes totales anuales se ajustan a una función de distribución doble Gumbel (González, 1970) en la que la población mayor corresponde a años con eventos extraordinarios de invierno, se modificó el procedimiento de selección de los años, de forma que, si el valor total anual sintético generado es mayor que un umbral que separa las dos poblaciones, 
DOI: http://dx.doi.org/10.22201/fi.25940732e.2009.10n4.032

Cálculo de registros sintéticos de ingresos por cuenca propia de un sistema de presas de la región Noroeste de México, caracterizada por eventos invernales

la selección se hace sólo entre los años que pertenecen a la segunda población y en caso contrario entre los de la primera.

\section{Aplicación}

Se aplicó el método de Svanidze modificado a los datos históricos de los volúmenes de ingreso mensuales por cuenca propia de las presas Lázaro Cárdenas (Angostura), Son., Abraham González, Chih., Plutarco Elías
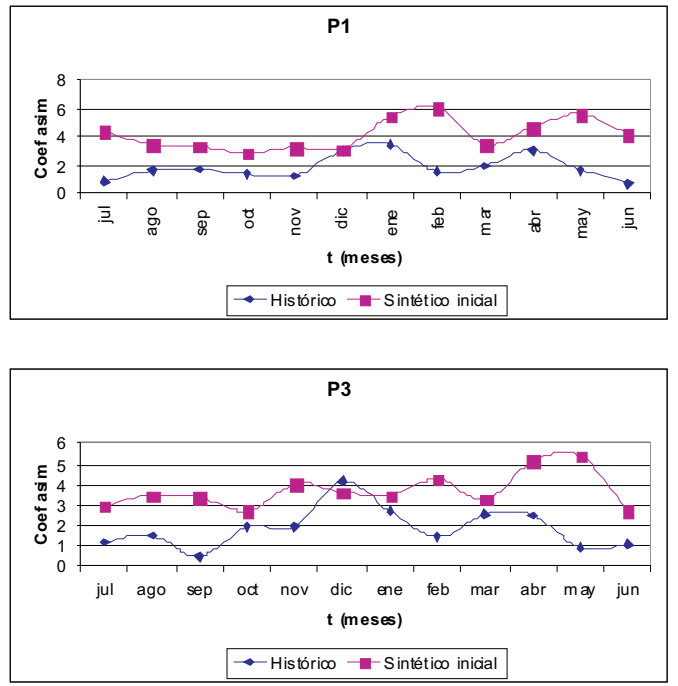

Calles (E1 Novillo), Son., y Álvaro Obregón (Oviachic), Son., utilizando los datos del funcionamiento mensual de las presas obtenidos del Banco Nacional de Datos de Aguas Superficiales (BANDAS) de la Comisión Nacional del Agua. Se formó un archivo de 26 años no continuos (1964-1966, 1968-1985 y 1995-1999) de escurrimientos simultáneos mensuales por cuenca propia (es decir, restando a las entradas totales las descargas de las presas situadas aguas arriba) para cada una de las presas. Para preparar los archivos de entrada al algoritmo se
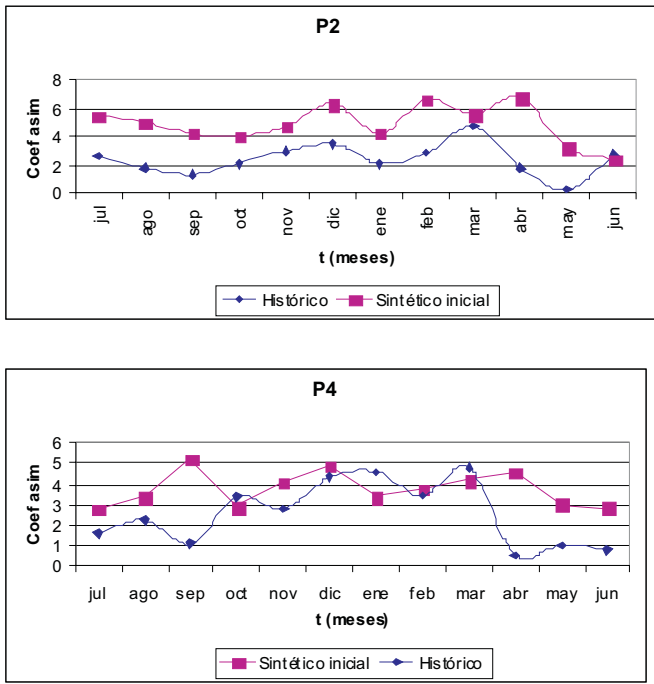

Figura 3. Coeficientes de asimetría obtenidos con el método de Svanidze en su forma original

Tabla 1. Serie mensual de la suma de las 4 presas analizadas (Ingresos en $\mathrm{hm}^{3}$ ). Estadísticos básicos

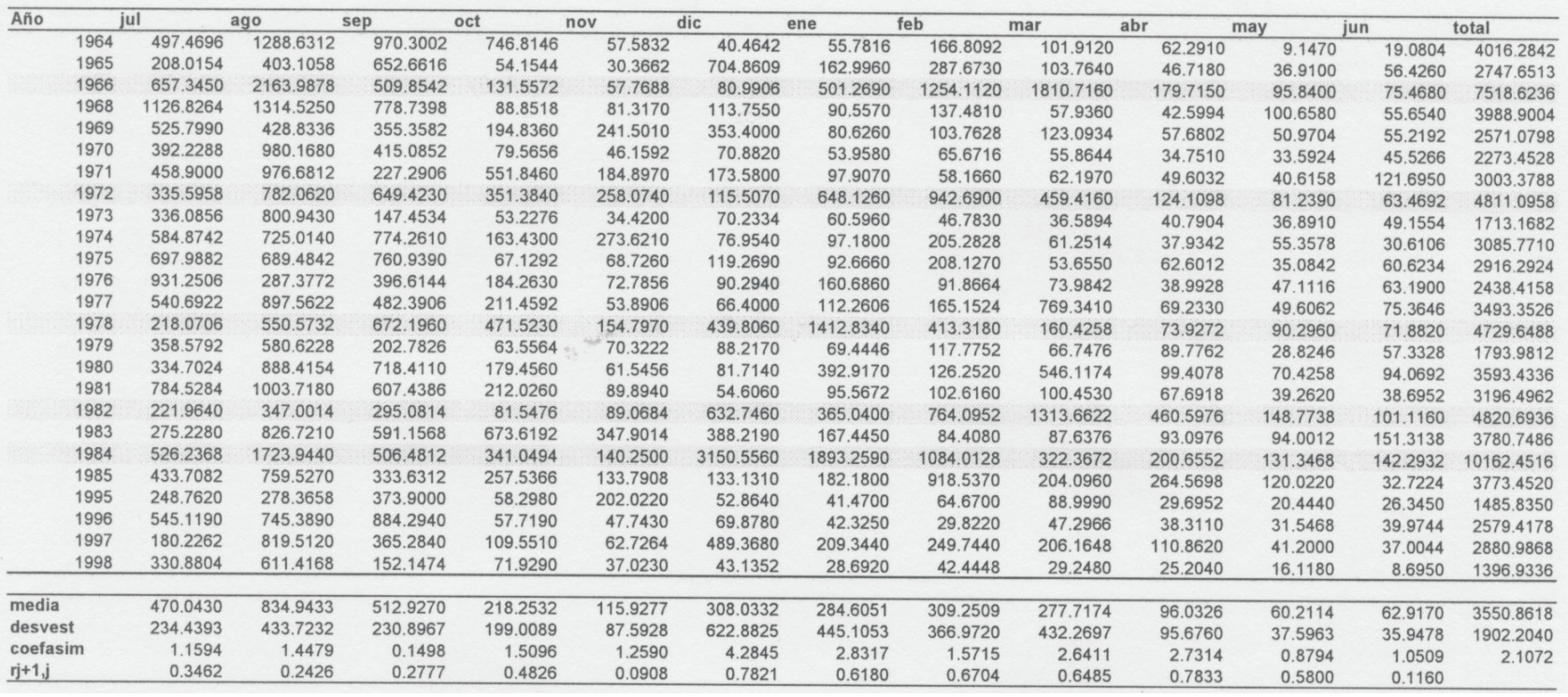


DOI: http://dx.doi.org/10.22201/fi.25940732e.2009.10n4.032

R. Domínguez-Mora y M.L. Arganis-Juárez

identificaron los meses de más baja correlación, a partir del registro construido con la suma de las 4 presas (tabla 1). De acuerdo con la tabla 1, la menor correlación se tiene entre el mes de junio y el de julio, por lo que se decidió trabajar con un año hidrológico, cuyo mes inicial es julio y el mes final es junio (tabla 2).

\section{Resultados}

Al realizar el análisis estadístico del total suma, con ayuda del sofware AX (Jiménez, 1996), se obtuvo que la función a la que mejor se ajustaban los datos fue una función de distribución Doble Gumbel (González, 1970), (Aparicio, 1989) con parámetros $\alpha_{1}=0.001269$ $\beta_{1}=255.0534 \alpha_{2}=0.000309 \quad \beta_{2}=6111.5934 p=0.85$; el valor de $p$ indica que el $85 \%$ de los datos corresponden a una primera población y el $15 \%$ restante a una segunda población. Al ordenar los eventos de mayor a menor se identificaron cinco años con ingresos extraordinarios en invierno (figura 4).

Se generaron 1000 datos sintéticos del total suma; la figura 5, muestra tanto los datos sintéticos como los datos históricos y calculados, dibujados en escala Gumbel.

Por otro lado, se obtuvieron 1000 años seleccionados aleatoriamente (con reemplazo) de entre los datos de la muestra histórica para considerar los porcentajes con respecto al valor total suma, correspondientes a cada presa, así como sus respectivas fracciones mensuales. Para ello, se comparó el ingreso total suma sintético con respecto a 4729 (quinto valor de la figura 4), de tal forma que si el valor sintético era superior a dicho valor, la selección aleatoria se hacía entre los años de mayor valor (en este caso los años 1966, 1972, 1982, 1984), en caso contrario la selección aleatoria se hacía entre los años restantes.

En la figura 5 se presenta la comparación de los ingresos por cuenca propia total anual (suma de las cuatro presas) histórico y sintético; se calcularon los estadísticos media, desviación estándar, coeficiente de asimetría, coeficiente de autocorrelación y de correlación cruzada; la comparación entre los estadísticos históricos y calculados se muestra en las Figuras 6 a 10; en las que se denota como P1 a Lázaro cárdenas (Angostura), P2 a Abraham González, P3 a Plutarco E Calles (El Novillo) y P4 a Álvaro Obregón (Oviachic).

Tabla 2. Año hidrológico, suma de las 4 presas analizadas. Estadísticos básicos

\begin{tabular}{|c|c|c|c|c|c|c|c|c|c|c|c|c|c|}
\hline Año & ene & feb & mar & $a b r$ & may & jun & jul & ago & sep & oct & nov & dic & Total \\
\hline 1964 & 68.9898 & 57.7900 & 78.8598 & 38.7372 & 43.5102 & 16.5046 & 497.4696 & 1288.6312 & 970.3002 & 746.8146 & 57.5832 & 40.4642 & 3905.6546 \\
\hline 1965 & 55.7816 & 166.8092 & 101.9120 & 62.2910 & 9.1470 & 19.0804 & 208.0154 & 403.1058 & 652.6616 & 54.1544 & 30.3662 & 704.8609 & 2468.1855 \\
\hline 1966 & 162.9960 & 287.6730 & 103.7640 & 46.7180 & 36.9100 & 56.4260 & 657.3450 & 2163.9878 & 509.8542 & 131.5572 & 57.7688 & 80.9906 & 4295.9906 \\
\hline 1968 & 501.2690 & 1254.1120 & 1810.7160 & 179.7150 & 95.8400 & 75.4680 & 1126.8264 & 1314.5250 & 778.7398 & 88.8518 & 81.3170 & 113.7550 & 7421.1350 \\
\hline 1969 & 90.5570 & 137.4810 & 57.9360 & 42.5994 & 100.6580 & 55.6540 & 525.7990 & 428.8336 & 355.3582 & 194.8360 & 241.5010 & 353.4000 & 2584.6132 \\
\hline 1970 & 80.6260 & 103.7628 & 123.0934 & 57.6802 & 50.9704 & 55.2192 & 392.2288 & 980.1680 & 415.0852 & 79.5656 & 46.1592 & 70.8820 & 2455.4408 \\
\hline 1971 & 53.9580 & 65.6716 & 55.8644 & 34.7510 & 33.5924 & 45.5266 & 458.9000 & 976.6812 & 227.2906 & 551.8460 & 184.8970 & 173.5800 & 2862.5588 \\
\hline 1972 & 97.9070 & 58.1660 & 62.1970 & 49.6032 & 40.6158 & 121.6950 & 335.5958 & 782.0620 & 649.4230 & 351.3840 & 258.0740 & 115.5070 & 2922.2298 \\
\hline 1973 & 648.1260 & 942.6900 & 459.4160 & 124.1098 & 81.2390 & 63.4692 & 336.0856 & 800.9430 & 147.4534 & 53.2276 & 34.4200 & 70.2334 & 3761.4130 \\
\hline 1974 & 60.5960 & 46.7830 & 36.5894 & 40.7904 & 36.8910 & 49.1554 & 584.8742 & 725.0140 & 774.2610 & 163.4300 & 273.6210 & 76.9540 & 2868.9594 \\
\hline 1975 & 97.1800 & 205.2828 & 61.2514 & 37.9342 & 55.3578 & 30.6106 & 697.9882 & 689.4842 & 760.9390 & 67.1292 & 68.7260 & 119.2690 & 2891.1524 \\
\hline 1976 & 92.6660 & 208.1270 & 53.6550 & 62.6012 & 35.0842 & 60.6234 & 931.2506 & 287.3772 & 396.6144 & 184.2630 & 72.7856 & 90.2940 & 2475.3416 \\
\hline 1977 & 160.6860 & 91.8664 & 73.9842 & 38.9928 & 47.1116 & 63.1900 & 540.6922 & 897.5622 & 482.3906 & 211.4592 & 53.8906 & 66.4000 & 2728.2258 \\
\hline 1978 & 112.2606 & 165.1524 & 769.3410 & 69.2330 & 49.6062 & 75.3646 & 218.0706 & 550.5732 & 672.1960 & 471.5230 & 154.7970 & 439.8060 & 3747.9236 \\
\hline 1979 & 1412.8340 & 413.3180 & 160.4258 & 73.9272 & 90.2960 & 71.8820 & 358.5792 & 580.6228 & 202.7826 & 63.5564 & 70.3222 & 88.2170 & 3586.7632 \\
\hline 1980 & 69.4446 & 117.7752 & 66.7476 & 89.7762 & 28.8246 & 57.3328 & 334.7024 & 888.4154 & 718.4110 & 179.4560 & 61.5456 & 81.7140 & 2694.1454 \\
\hline 1981 & 392.9170 & 126.2520 & 546.1174 & 99.4078 & 70.4258 & 94.0692 & 784.5284 & 1003.7180 & 607.4386 & 212.0260 & 89.8940 & 54.6060 & 4081.4002 \\
\hline 1982 & 95.5672 & 102.6160 & 100.4530 & 67.6918 & 39.2620 & 38.6952 & 221.9640 & 347.0014 & 295.0814 & 81.5476 & 89.0684 & 632.7460 & 2111.6940 \\
\hline 1983 & 365.0400 & 764.0952 & 1313.6622 & 460.5976 & 148.7738 & 101.1160 & 275.2280 & 826.7210 & 591.1568 & 673.6192 & 347.9014 & 388.2190 & 6256.1302 \\
\hline 1984 & 167.4450 & 84.4080 & 87.6376 & 93.0976 & 94.0012 & 151.3138 & 526.2368 & 1723.9440 & 506.4812 & 341.0494 & 140.2500 & 3150.5560 & 7066.4206 \\
\hline 1985 & 1893.2590 & 1084.0128 & 322.3672 & 200.6552 & 131.3468 & 142.2932 & 433.7082 & 759.5270 & 333.6312 & 257.5366 & 133.7908 & 133.1310 & 5825.2590 \\
\hline 1995 & 182.1800 & 918.5370 & 204.0960 & 264.5698 & 120.0220 & 32.7224 & 248.7620 & 278.3658 & 373.9000 & 58.2980 & 202.0220 & 52.8640 & 2936.3390 \\
\hline 1996 & 41.4700 & 64.6700 & 88.9990 & 29.6952 & 20.4440 & 26.3450 & 545.1190 & 745.3890 & 884.2940 & 57.7190 & 47.7430 & 69.8780 & 2621.7652 \\
\hline 1997 & 42.3250 & 29.8220 & 47.2966 & 38.3110 & 31.5468 & 39.9744 & 180.2262 & 819.5120 & 365.2840 & 109.5510 & 62.7264 & 489.3680 & 2255.9434 \\
\hline 1998 & 209.3440 & 249.7440 & 206.1648 & 110.8620 & 41.2000 & 37.0044 & 330.8804 & 611.4168 & 152.1474 & 71.9290 & 37.0230 & 43.1352 & 2100.8510 \\
\hline 1999 & 28.6920 & 42.4448 & 29.2480 & 25.2040 & 16.1180 & 8.6950 & 940.0030 & 924.4140 & 359.8080 & 52.1526 & 20.8250 & 36.8768 & 2484.4812 \\
\hline media & 276.3122 & 299.5793 & 270.0690 & 93.8289 & 59.5690 & 61.1319 & 488.1184 & 838.3844 & 507.0378 & 211.8647 & 112.2699 & 297.6041 & 3515.7699 \\
\hline & 438.1576 & 362.9238 & 425.3278 & 94.4140 & 36.9821 & 36.3786 & 247.5036 & 425.3223 & 228.2160 & 197.6904 & 87.8263 & 612.6102 & 1506.1934 \\
\hline coefasim & 2.8966 & 1.6267 & 2.7068 & 2.7858 & 0.9365 & 1.0398 & 1.0016 & 1.4431 & 0.2192 & 1.5528 & 1.2754 & 4.3607 & 1.5477 \\
\hline $\mathrm{rj}+1, \mathrm{j}$ & 0.6225 & 0.6739 & 0.6521 & 0.7852 & 0.5815[ & -0.0165 & 0.3364 & 0.2348 & 0.2932 & 0.5001 & 0.1068 & 0.4282 & \\
\hline
\end{tabular}




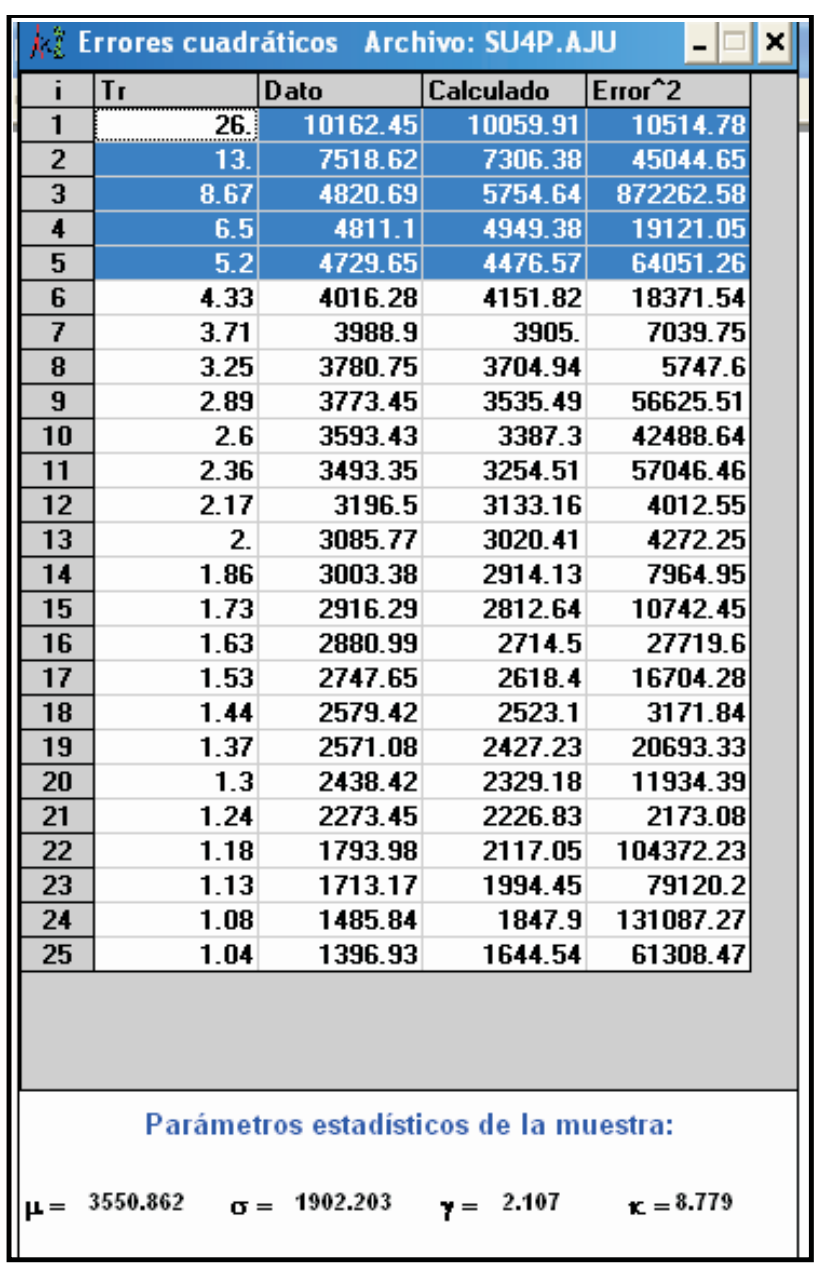

Figura 4. Datos históricos y calculados
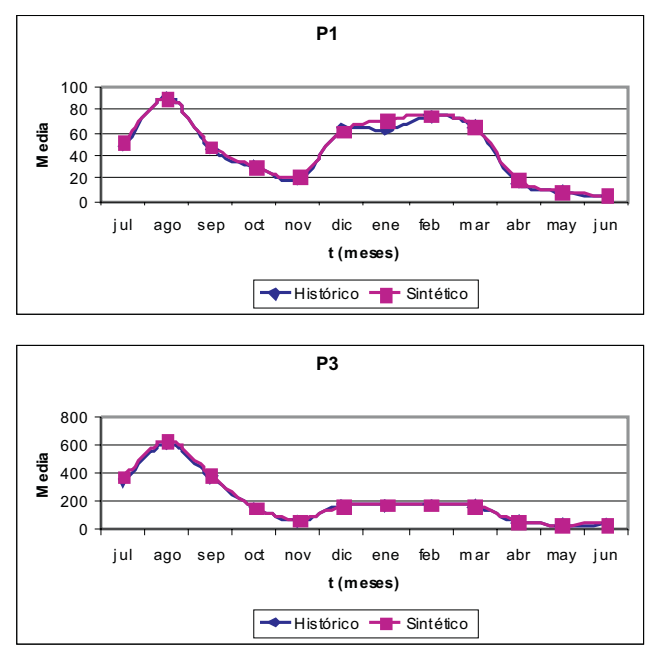

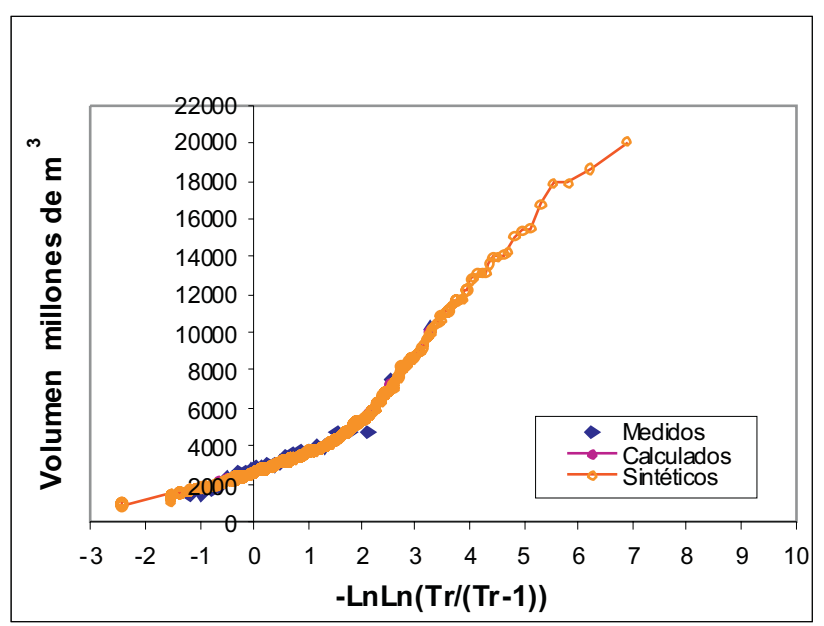

Figura 5. Función Doble Gumbel, datos históricos y calculados. 1000 datos sintéticos generados
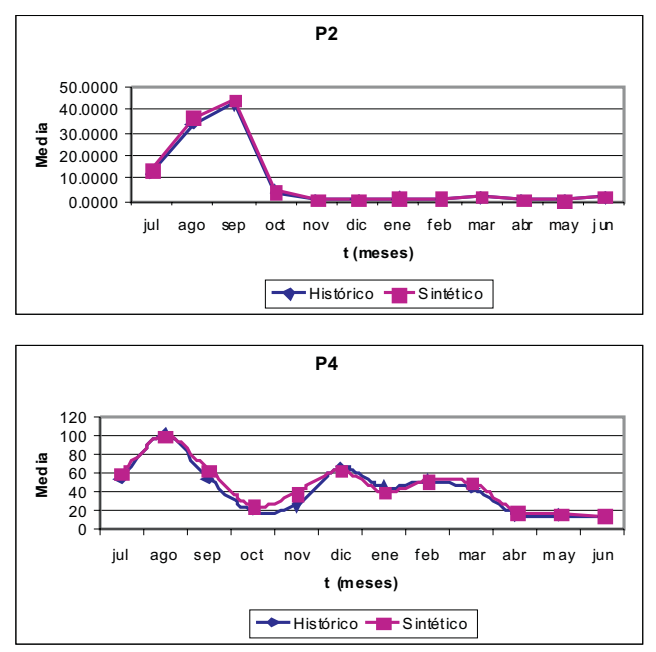

Figura 6. Comparación de las medias $\left(\mathrm{hm}^{3}\right)$ 

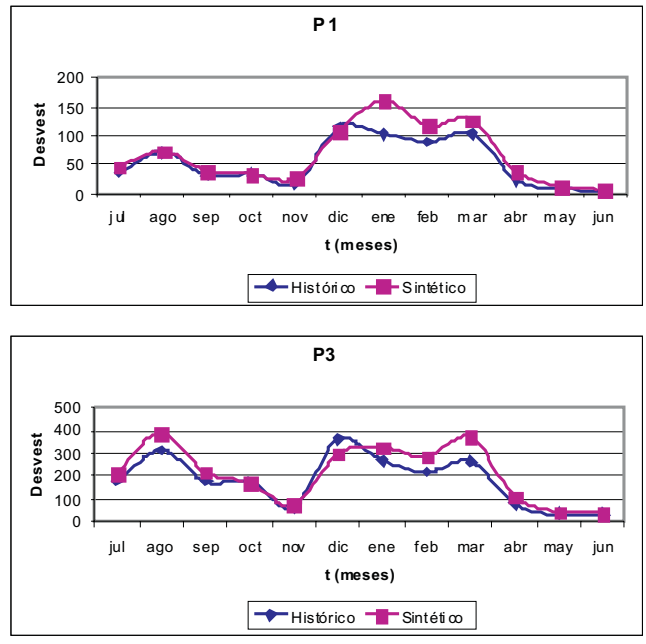
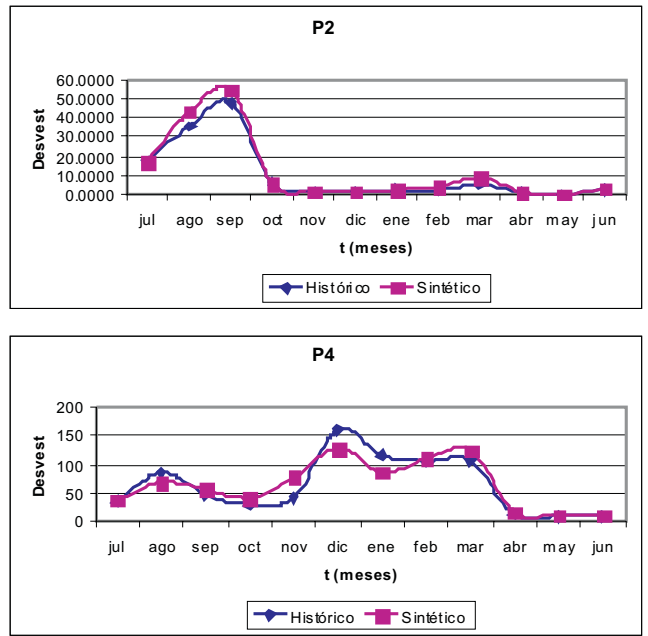

Figura 7. Comparación de las desviaciones estándar ( $\left.\mathrm{hm}^{3}\right)$
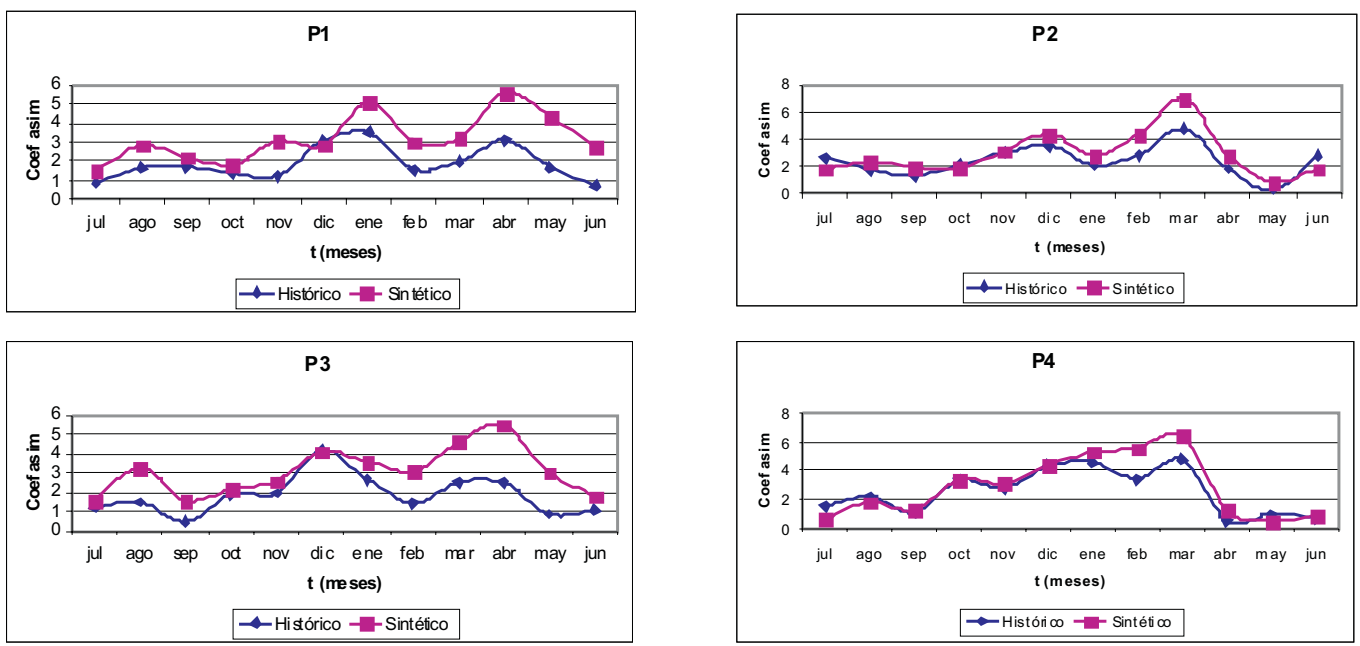

Figura 8. Comparación de los coeficientes de asimetría 
DOI: http://dx.doi.org/10.22201/fi.25940732e.2009.10n4.032

Cálculo de registros sintéticos de ingresos por cuenca propia de un sistema de presas de la región Noroeste de México, caracterizada por eventos invernales
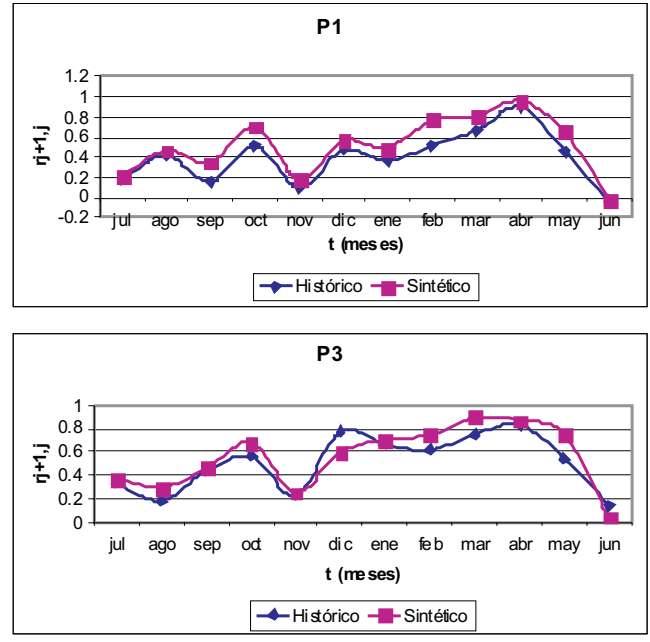
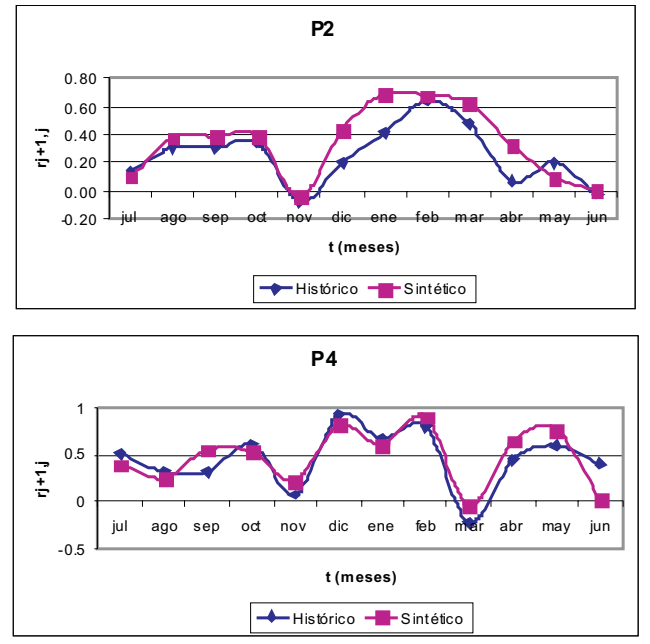

Figura 9. Comparación de los coeficientes de autocorrelación
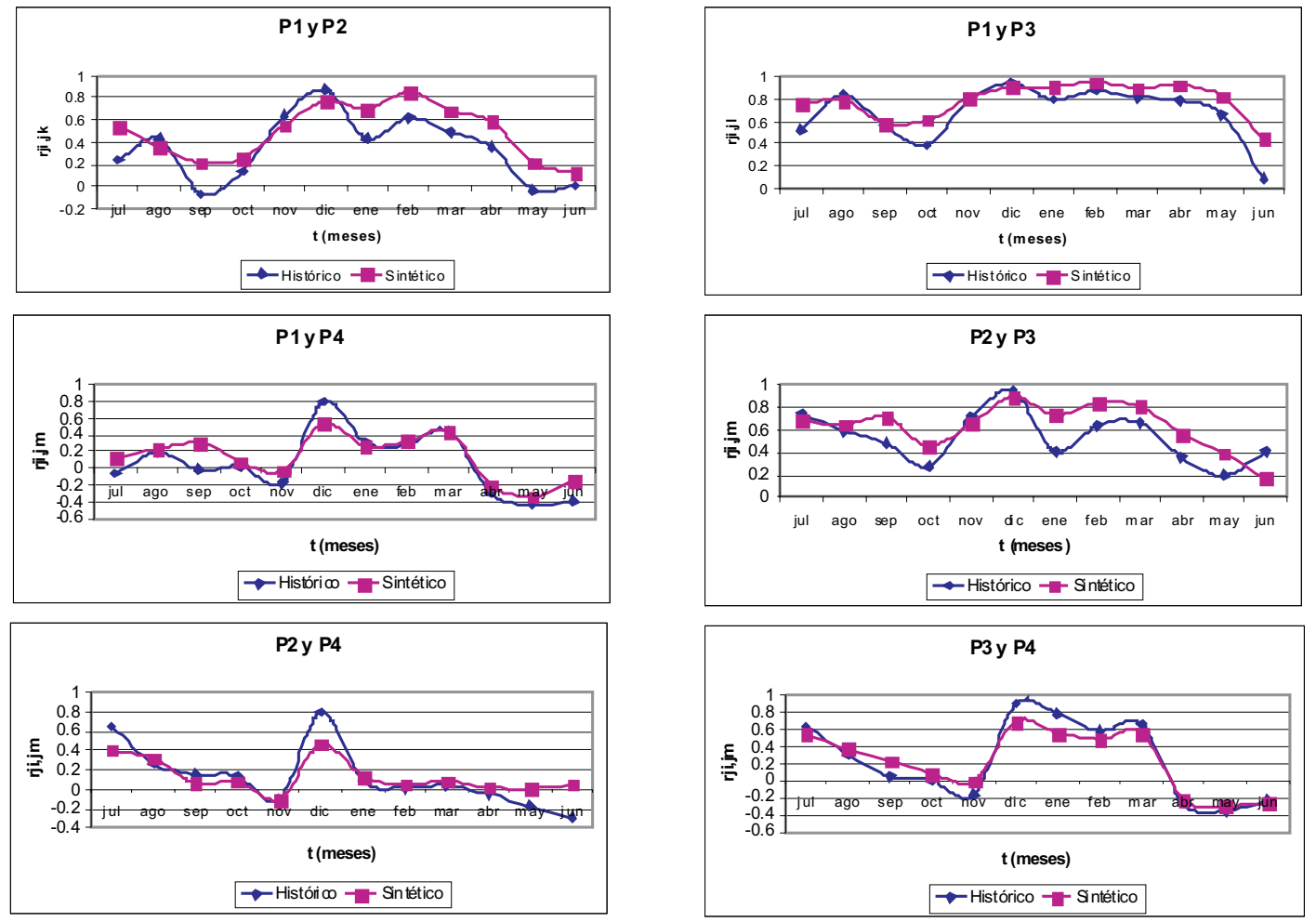

Figura 10. de los coeficientes de correlación cruzada 
DOI: http://dx.doi.org/10.22201/fi.25940732e.2009.10n4.032

R. Domínguez-Mora y M.L. Arganis-Juárez

\section{Conclusiones}

De acuerdo con los resultados obtenidos se puede concluir que el método de Svanidze modificado en la selección de años aleatorios es una herramienta útil y práctica en la determinación de muestras sintéticas de los ingresos por cuenca propia de un sistema de presas conectadas. Los estadísticos obtenidos preservan adecuadamente los de las series históricas; en particular, cabe destacar una muy buena concordancia entre los coeficientes de asimetría obtenidos al modificar la selección de los años aleatorios; se preservaron las autocorrelaciones y las correlaciones cruzadas, lo que no sucedía al aplicar al método en su forma tradicional a series de tiempo caracterizadas por estar sujetas a eventos extraordinarios de tipo invernal.

\section{Agradecimientos}

Se agradece a la M. I. Gabriela Esquivel Garduño su colaboración en el diseño de imágenes en ambiente Arc View.

\section{Referencias}

Aparicio M.F.J. Fundamentos de hidrología de superficie. México. Limusa. 1989.
Domínguez M.R., Fuentes M.G. y Arganis J.M.L. Procedimiento para generar muestras sintéticas de series periódicas mensuales a través del Método de Svanidze modificado aplicado a los datos de las presas La Angostura y Malpaso. Series Instituto de Ingeniería. C1-19. 2001.

Domínguez M.R., Fuentes M.G.E. y Arganis J.M.L. Generación de muestras sintéticas de volúmenes de escurrimiento mensual de las presas La Angostura y Malpaso, Chiapas, utilizando el Método de Svanidze Modificado. XX Congreso Latinoamericano de Hidráulica., Ciudad de La Habana. 2002.

Escalante S.C.A y Reyes C.L. Técnicas estadísticas en hidrología. México. Facultad de Ingeniería, UNAM. 2002.

González V.F.J. Contribución al análisis de frecuencias de valores extremos de los gastos máximos en un río. México. Instituto de Ingeniería. Serie Azul. No. 277. 1970.

Jiménez E.M. Programa AX. Área de riesgos hidrometeorológicos. Centro Nacional de Prevención de Desastres. México. 1996,

Salas J.D., Delleur J.W., Yevjevich y Lane W.L. Applied Modeling of Hydrological Time Series. USA. Water Resources Publications. 1988.

Svanidze G.G. Mathematical Modeling of Hidrologic Series. USA. Water Resources Publications. 1980.

Yurekly K., Kurunk A. y Simsek H. Prediction of Daily Maximum Streamflow Based on Stochastic Approaches. Journal of Spatial Hydrology, 4(2), 2004

\section{Semblanza de los autores}

Ramón Dominguez-Mora. Egresado de la Facultad de Ingeniería, UNAM con maestría y doctorado. Se desempeña como investigador titular en la Coordinación de Hidráulica del Instituto de Ingeniería de la UNAM, así como profesor del Posgrado de Ingeniería de la misma institución. Ha realizado amplios trabajos en material de hidrología superficial, hidrología urbana y aprovechamientos hidráulicos.

Maritza L. Arganis-Juárez. Es egresada de la Facultad de Ingeniería, UNAM con maestría y doctorado. Actualmente es investigador asociado de la Coordinación de Hidráulica del Instituto de Ingeniería de la UNAM y profesora de asignatura definitiva de la Facultad de Ingeniería, UNAM. En 1993, inició su colaboración con esta institución como profesora de física en la Escuela Nacional Preparatoria; actualmente realiza investigaciones en el área de aprovechamientos hidráulicos e hidrología. 